\title{
GEOMETRIES OF PATHS FOR WHICH THE EQUATIONS OF THE PATHS ADMIT A QUADRATIC FIRST INTEGRAL*
}

BY

\section{LUTHER PFAHLER EISENHART}

1. A geometry of paths for a general space of $n$ dimensions is based upon the conception that the paths are fundamental entities of the space. They are the integral curves of a system of differential equations

$$
\frac{d^{2} x^{i}}{d s^{2}}+\Gamma_{j k}^{i} \frac{d x^{j}}{d s} \frac{d x^{k}}{d s}=0
$$

where $x^{i}(i=1, \cdots, n)$ are the coordinates of a point, $\Gamma_{j k}^{i}\left(=\Gamma_{k j}^{i}\right)$ are functions of the $x$ 's and $s$ is a parameter peculiar to each path. An important class of these geometries, which admits the Riemannian geometry as a sub-class, is that for which the equations (1.1) admit a quadratic first integral

$$
g_{i j} \frac{d x^{i}}{d s} \frac{d x^{j}}{d s}=\text { const. }
$$

where $g_{i j}\left(=g_{j i}\right)$ are the components of a covariant tensor. Veblen and Thomast have considered the problem, given a set of $I$ 's to determine whether equations (1.1) admit an integral (1.2); they have shown that its solution involves only algebraic processes. In this paper the converse problem is solved, namely to determine the $\Gamma$ 's so that (1.1) shall admit a given first integral (1.2); also the more general problem when the first integral is of the form

$$
e^{\int \varphi_{\alpha} d x^{\alpha}} g_{i j} \frac{d x^{i}}{d s} \frac{d x^{j}}{d s}=\text { const., }
$$

where $\varphi_{\alpha}$ is a vector and the integral is taken along the path.

2. General formulas. If we put $x^{i}=\varphi^{i}\left(x^{1}, \cdots, x^{\prime n}\right)$, thus introducing a new set of coordinates, equations (1.1) become

* Presented to the Society, March 1, 1924.

†These Transactions, vol. 25 (1923), pp. 599-608. 


$$
\frac{d^{2} x^{\prime i}}{d s^{2}}+\Gamma_{\alpha \beta}^{\prime i} \frac{d x^{\prime \alpha}}{d s} \frac{d x^{\prime \beta}}{d s}=0,
$$

where

$$
\frac{\partial^{2} x^{k}}{\partial x^{\prime \alpha} \partial x^{\prime \beta}}+\Gamma_{i j}^{k} \frac{\partial x^{i}}{\partial x^{\prime \alpha}} \frac{\partial x^{j}}{\partial x^{\prime \beta}}=\Gamma_{\alpha \beta}^{\prime \sigma} \frac{\partial x^{k}}{\partial x^{\prime \sigma}}
$$

Suppose now that $g_{i j}$ are the components of a symmetric covariant tensor of the second order, that is

$$
g_{\alpha \beta}^{\prime}=g_{i j} \frac{\partial x^{i}}{\partial x^{\prime \alpha}} \frac{\partial x^{j}}{\partial x^{\prime \beta}},
$$

then by means of (2.2) it can be shown that the functions $g_{i j, k}$, defined by

$$
g_{i j, k}=\frac{\partial g_{i j}}{\partial x^{k}}-g_{i \alpha} \Gamma_{j k}^{\alpha}-g_{\alpha j} \Gamma_{i k}^{\alpha}
$$

are the components of a covariant tensor of the third order. As thus defined $g_{i j, k}$ is a generalization of the first covariant derivative of $g_{i j}$, the ordinary covariant derivative for a Riemann space being given by (2.4) when $\Gamma_{j k}^{\alpha}$ is replaced by $\left\{\begin{array}{c}\alpha \\ j k\end{array}\right\}$, the Christoffel symbol of the second kind formed with respect to the fundamental form of the space,* and similarly for $\Gamma_{i k}^{\alpha}$.

The components $g^{i j}$ of the contravariant tensor associate to $g_{i j}$ are given by

$$
g^{i \alpha} g_{j \alpha}=\delta_{j}^{i}
$$

where

$$
\delta_{j}^{i}=0 \text { or } 1, \text { as } i \neq j \text { or } i=j
$$

As thus defined, $g^{i j}$ is the cofactor of $g_{i j}$ in the determinant $g=\left|g_{i j}\right|$ divided by $g$.

If we put

$$
[i j, k]=\frac{1}{2}\left(\frac{\partial g_{i k}}{\partial x^{j}}+\frac{\partial g_{j k}}{\partial x^{i}}-\frac{\partial g_{i j}}{\partial x^{k}}\right)
$$

and

$$
\left\{\begin{array}{l}
k \\
i j
\end{array}\right\}=g^{k \alpha}[i j, \alpha]
$$

* Cf. Proceedings of the National Academy of Sciences, vol. 8 (1922), p. 21. 
then $[i j, k]$ and $\left\{\begin{array}{c}k \\ i j\end{array}\right\}$ are the Christoffel symbols of the first and second kinds respectively formed with respect to the $g$ 's.

If we write also

$$
\Gamma_{j k, i}=g_{\alpha i} \Gamma_{j k}^{\alpha}, \quad \Gamma_{j k}^{\alpha}=g^{\alpha i} \Gamma_{j k, i},
$$

then from (2.4) and simular equations we have

$$
g_{k i, j}+g_{j k, i}-g_{i j, k}=2\left([i j, k]-\Gamma_{i j, k}\right)
$$

From (2.3) we have by differentiation and suitable operations*

$$
\frac{\partial^{2} x^{k}}{\partial{x^{\prime}}^{\alpha} \partial x^{\prime \beta}}+\left\{\begin{array}{c}
k \\
i j
\end{array}\right\} \frac{\partial x^{i}}{\partial x^{\prime \alpha}} \frac{\partial x^{j}}{\partial x^{\prime \beta}}=\left\{\begin{array}{c}
\sigma \\
\alpha \beta
\end{array}\right\}^{\prime} \frac{\partial x^{k}}{\partial x^{\prime \sigma}}
$$

where $\left\{\begin{array}{c}\sigma \\ \alpha \beta\end{array}\right\}$ is formed with respect to the $g^{\prime \prime}$ s. Subtracting this equation from (2.2), we obtain

$$
\Gamma_{\alpha \beta}^{\prime \sigma}-\left\{\begin{array}{c}
\sigma \\
\alpha \beta
\end{array}\right\}^{\prime}=\left(\Gamma_{i j}^{k}-\left\{\begin{array}{c}
k \\
i j
\end{array}\right\}\right) \frac{\partial x^{i}}{\partial x^{\prime \alpha}} \frac{\partial x^{j}}{\partial x^{\prime \beta}} \frac{\partial x^{\prime \sigma}}{\partial x^{k}} .
$$

Hence if we put

$$
\Gamma_{i j}^{k}=\left\{\begin{array}{l}
k \\
i j
\end{array}\right\}+c_{i j}{ }^{k}
$$

the functions $c_{i j}{ }^{k}$ are the components of a tensor contravariant of the first order and covariant (and symmetric) of the second order. In consequence of (2.8) and (2.9) we have from (2.12)

$$
\boldsymbol{\Gamma}_{i j, k}=[i j, k]+c_{i j k} .
$$

Hence (2.10) becomes

$$
g_{k i, j}+g_{j k, i}-g_{i j, k}=-2 c_{i j k} \text {. }
$$

If we add to this equation the two equations obtained from it by permuting $i, j, k$ cyclically, we obtain

$$
g_{i j, k}+g_{j k, i}+g_{k i, j}=-2\left(c_{i j k}+c_{j k i}+c_{k i j}\right) .
$$

\footnotetext{
* Cf. Bianchi, Lezioni, vol. 1, p. 64.
} 
3. Quadratic first integrals. When we express the condition that (1.2) be a first integral of (1.1), we obtain

$$
g_{i j, k} \frac{d x^{i}}{d s} \frac{d x^{j}}{d s} \frac{d x^{k}}{d s}=0
$$

Since this must be satisfied identically, we must have

$$
g_{i j, k}+g_{j k, i}+g_{k i, j}=0 \quad(i, j, k=1, \cdots, n)
$$

The consistency of these equations is the necessary and sufficient condition that (1.2) be a first integral.*

We consider also the case when (1.1) admits a first integral of the form

$$
e^{\int \varphi_{\alpha} d x^{\alpha}} g_{i j} \frac{d x^{i}}{d s} \frac{d x^{j}}{d s}=\text { const., }
$$

where $\varphi_{\alpha}$ are the components of a vector and the integral $\int \varphi_{\alpha} d x^{\alpha}$ is taken along the path in question. Proceeding as above, we obtain the equations

$$
g_{i j, k}+g_{j k, i}+g_{k i, j}+g_{i j} \varphi_{k}+g_{j k} \varphi_{i}+g_{k i} \varphi_{j}=0
$$

Conversely, if these equations are consistent and yield a tensor $g_{i j}$ and a vector $\varphi_{\alpha}$, then (3.2) is a first integral of the corresponding equations (1.1). It can be shownt that if we put

$$
\bar{\Gamma}_{i j}^{k}=\Gamma_{i j}^{k}-\frac{1}{4}\left(\delta_{i}^{k} \varphi_{j}+\delta_{j}^{k} \varphi_{i}\right)
$$

and define a parameter $\bar{s}$ for each path by means of the equation

$$
\frac{d \bar{s}}{d s}=e^{-\frac{1}{2} \int \varphi_{\alpha} d x^{\alpha}}
$$

* Veblen and Thomas, loc. cit., pp. 599-608, give a complete treatment of the question of the consistency of equations (3.1).

† Annals of Mathematics, ser. 2, vol. 24 (1923), p. 376. 
the equations

$$
\frac{d^{2} x^{k}}{d \bar{s}^{2}}+\bar{\Gamma}_{i j}^{k} \frac{d x^{i}}{d \bar{s}} \frac{d x^{j}}{d \bar{s}}=0
$$

define the same curves as (1.1).

If we denote by $g_{i j, \bar{k}}$ the covariant derivative of $g_{i j}$ given by the equation obtained by replacing $\Gamma_{i j}^{k}$ by $\bar{\Gamma}_{i j}^{k}$ in (2.4), we have

$$
g_{i j, \bar{k}}=g_{i j, k}+\frac{1}{4}\left(2 g_{i j} \varphi_{k}+g_{j k} \varphi_{i}+g_{k i} \varphi_{j}\right) .
$$

Now (3.3) becomes

$$
g_{i j, \bar{k}}+g_{j k, \bar{i}}+g_{k i, \bar{j}}=0,
$$

and (3.2) reduces to the form (1.2) by means of (3.5).

The change (3.4) in the $\Gamma$ 's means a change in the affine connection of the space, but not in the paths themselves. Hence we have the theorem

When the equations of the paths of a space admit a first integral of the form (3.2), by a change in the affine connection but not in the paths themselves the equations of the paths can be given a form which admits a first integral of the form (1.2).

When, in particular,

$$
g_{i j, k}=-g_{i j} \varphi_{k}
$$

equations (3.3) are satisfied. This is Weyl's geometry, for it follows from (2.10) that

$$
\Gamma_{i j, k}=[i j, k]+\frac{1}{2}\left(g_{j k} \varphi_{i}+g_{k i} \varphi_{j}-g_{i j} \varphi_{k}\right) . *
$$

Consequently, if the paths are taken as fundamental rather than the affine connection, it follows from (3.4) that if in (1.1) we take

$$
\Gamma_{i j}^{k}=\left\{\begin{array}{c}
k \\
i j
\end{array}\right\}+\frac{1}{4}\left(\delta_{j}^{k} \varphi_{i}+\delta_{i}^{k} \varphi_{j}-g_{i j} \varphi^{k}\right)
$$

we have a geometry of the space with the same paths, whose equations admit the first integral (1.2).

Returning to the consideration of (3.1), we observe that from this equation and (2.15) we have the theorem

* Cf. Space, Time and Matter, p. 125. 
A necessary and sufficient condition that equations (1.1) admit a quadratic first integral (1.2) is that the tensor $c_{i j k}$ defined by (2.13) satisfy the conditions

$$
c_{i j k}+c_{j k i}+c_{k i j}=0
$$

4. Determination of geometries of paths with quadratic first integrals. Suppose that we have any symmetric tensor $g_{i j}$ and a covariant tensor of the third order, $a_{i j k}$, symmetric in $i$ and $j$. If we define a tensor, $c_{i j k}$, by means of the equations

$$
c_{i j k}=2 a_{i j k}-a_{i k j}-a_{j k i} \quad(i, j, k=1, \cdots, n),
$$

it is by definition symmetric in $i$ and $j$, and satisfies (3.12). If we define a set of $\Gamma$ 's by means of (2.13) in which $[i j, k]$ are formed with respect to the given tensor $g_{i j}$ and $c_{i j k}$ is given by (4.1), it follows from the theorem at the end of $\S 3$ that the equations (1.1) admit the corresponding first integral (1.2).

Consider, conversely, the case when a geometry of this kind is given. Then the $c_{i j k}$ as given by (2.13) satisfy (3.12). This condition is met, if we determine the components $a_{i j k}$ of a tensor such that (4.1) hold for $c_{i j k}$ known.

For each set of values of $i, j, k$ all different, there are two equations (4.1), which are equivalent to

$$
\begin{aligned}
& a_{i j k}-a_{k i j}=d_{j i k}, \\
& a_{j k i}-a_{k i j}=d_{j k i} \quad(i, j, k=1, \cdots, n ; i, j, k \neq),
\end{aligned}
$$

where

$$
d_{j i k}=\frac{1}{3}\left(2 c_{j i k}+c_{j k i}\right)=\frac{1}{3}\left(c_{j i k}-c_{k i j}\right),
$$

the second and third expressions being equivalent because of (3.12). From (4.3) follow the identities

$$
\begin{aligned}
d_{j i k}+d_{k i j} & =0 \\
d_{j i k}+d_{k j i}+d_{i k j} & =0 \quad(i, j, k=1, \cdots, n ; i, j, k \neq) .
\end{aligned}
$$

In the general case, that is when $c_{i j k}$ do not satisfy any conditions other than (3.12), there are, in consequence of $(4.4), n(n-1)(n-2) / 3$ independent equations of the type (4.2). 
From (4.1) and (3.12) we have also

$$
a_{i i j}-a_{i j i}=d_{i i j} \quad(i, j=1, \cdots, n ; i, j \neq),
$$

where

$$
d_{i i j}=\frac{1}{2} c_{i i j}=\frac{1}{3}\left(c_{i i j}-c_{j i i}\right) .
$$

In the general case there are $n(n-1)$ independent equations of this type. From (3.1) we have $g_{i i, i}=0$, so that from (2.10) we have $\Gamma_{i i i}=[i i, i]$ and consequently $c_{i i i}=0$. In this case (4.1) vanishes identically and consequently the components $a_{i i i}$ are not determined. Hence there are $n(n-1)(n+1) / 3$ independent equations for the determination of the $n^{2}(n+1) / 2$ components of $a_{i j k}$. Consequently there aren $(n+1)(n+2) / 6$ components arbitrary: they are one for each case where $i, j, k$ are different; one where two are different; and all of the type $a_{i i i}$. Hence we have the theorem

$A$ tensor $g_{i j}$ and a tensor $a_{i j k}$, symmetric in $i$ and $j$, determine a geometry of paths whose equations admit the corresponding first integral (1.2); conversely, if a geometry is given whose equations admit a first integral (1.2), $n(n+1)(n+2) / 6$ of the components $a_{i j k}$ are arbitrary and the others can be found directly.

Princeton University,

Princeton, N. J. 